\title{
FACTORES DE RIESGO ASOCIADOS A CARIES DE INFANCIA TEMPRANA SEVERA
}

\section{RISK FACTORS ASSOCIATED WITH SEVERE EARLY CHILDHOOD CARIES}

\author{
Kariem Noelia Sherezade Siquero-Vera ${ }^{1 a}$, Manuel Antonio Mattos-Vela ${ }^{2 b}$
}

\section{RESUMEN}

La Caries de Infancia Temprana (CIT) se considera un problema de salud pública; cuando no es tratada oportunamente, la enfermedad avanza a un nivel severo. Actualmente existen muchas investigaciones sobre prevalencia y factores de riesgo de CIT, pero escasa en el tema de Caries de Infancia Temprana Severa (CIT-S). El presente artículo de revisión evalúa los distintos factores que se han relacionado a CIT-S, en los últimos años, agrupándolos en tres grandes áreas: huésped, microorganismo y sustrato. Se realizó una búsqueda en la base de datos LILACS, Medline y Elseiver; a partir del año 2010 con las palabras clave Caries de Infancia Temprana Severa, factores de riesgo, Severe Early Childhood Caries y risk factors.

Palabras Clave: Caries Dental; Factores de Riesgo; Etiología (Fuente: DeCS BIREME)

\section{ABSTRACT}

Early Childhood Caries (ECC) is considered a public health problem; when it is not treated in a timely manner, the disease progresses to a severe level. Currently there are many studies on the prevalence and risk factors of ECC, but little on the issue of Severe Early Childhood Caries (S-ECC). The present review article evaluates the different factors that have been related to S-ECC, in recent years, grouped into three major areas: host, microorganism and substrate. A search was made in the LILACS, Medline and Elsevier database; from the year 2010 with the keywords Caries of Severe Early Childhood, risk factors, Severe Early Childhood Caries and risk factors

Keywords: Dental caries; risk factors; Etiology. (Source: MeSH NLM)

Recibido: 13 de junio de 2018

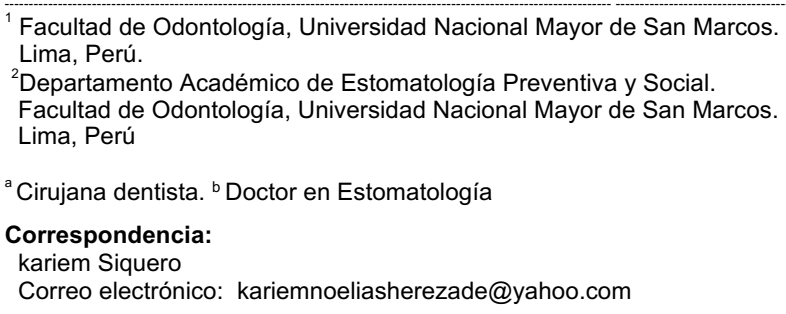

Este es un artículo Open Access distribuido bajo la licencia Creative Commons Atribución-NoComercial- Compartir Igual 4.0

\section{두)(웅}

Citar como: Siquero-Vera KNS, Mattos-Vela MA. Factores de riesgo asociados a caries de infancia temprana severa. Kiru. 2018; 15(3): 146-153. https://doi.org/10.24265/kiru.2018.v15n3.07 


\section{INTRODUCCIÓN}

La Organización Mundial de la Salud señala que la caries dental es el primer motivo de atención entre las enfermedades bucales ${ }^{(1)}$.

En la actualidad la Caries De Infancia Temprana (CIT) se considera un problema de salud pública, debido fundamentalmente a sus consecuencias sobre la salud de los niños y la economía de sus padres. Si se deja sin tratamiento, la CIT puede generar abscesos, dolor y maloclusión; así como alteraciones en el crecimiento y desarrollo normal del niño, hospitalizaciones y atenciones de urgencia; con lo cual hay un deterioro en la calidad de vida de los niños ${ }^{(2,3)}$.

Cuando la CIT no es tratada oportunamente, la enfermedad avanza a un nivel severo. Actualmente existen muchas investigaciones sobre prevalencia y factores de riesgo de CIT, pero escasa en el tema de Caries de Infancia Temprana Severa (CIT-S) ${ }^{(4,5)}$.

La literatura científica demuestra que ya durante el primer año de vida del bebé existe presencia de factores que aumentan el riesgo de aparición de caries dental, como los hábitos de higiene y de amamantamiento, entre otros. Gracias a la identificación de los factores de riesgo de caries dental se puede reconocer en cada infante individualmente en qué área se debe trabajar, eliminando o disminuyendo la influencia de este factor ${ }^{(6)}$.

En 1978, la Academia Americana de Odontología Pediátrica (AAPD) y la Asociación Dental Americana (ADA) publicaron una declaración en conjunto: "Jugo en biberones listos para usar y caries de biberón" para tratar una forma severa de caries asociada con el uso de estos, estableciendo recomendaciones iniciales limitadas a los hábitos alimenticios; sin embargo, en las dos décadas siguientes, se reconoció que la CIT no estaba únicamente asociada con las malas prácticas de alimentación, la AAPD adoptó el término CIT para reflejar mejor su etiología multifactorial. Estos factores incluyen dientes susceptibles debido a la hipoplasia del esmalte, colonización oral con niveles elevados de bacterias cariogénicas, especialmente Streptococcus mutans, y el metabolismo de azúcares por bacterias adherentes a los dientes para producir ácido que, con el tiempo, desmineraliza la estructura dental ${ }^{(7)}$.

Se han descrito los factores de riesgo de caries dental, muchos de ellos en el marco de la CIT ${ }^{(8-13)}$.

Los factores de riesgo que afectan a la CIT también se a la CIT-S; en esta revisión se describen estos factores dentro de los tres grandes grupos que juegan un papel importante en la Triada Etiológica de Keyes: huésped, microorganismo y sustrato ${ }^{(14,15)}$.

\section{Definición de CIT-S}

La ADA, el Centro de Prevención y Control de Enfermedades (CDC) de los EE.UU. y la AAPD definen a la CIT como cualquier tipo de lesión de caries presente en niños menores de 6 años o 71 meses y a la CIT-S cuando existen lesiones de caries en superficie lisa en un niño menor de tres años; en niños de tres a cinco años cuando hay piezas cavitadas, piezas faltantes (debido a caries) o piezas obturadas en superficies lisas de dientes anteriores maxilares primarios con una puntuación de cariada, ausente u obturada mayor o igual a cuatro (edad 3), mayor o igual a cinco (edad 4), mayor o igual a seis (edad 5$)^{(16)}$.

\section{Características clínicas}

La CIT representa el patrón de caries más precoz, agresivo y destructivo que existe, cuyo pico más elevado ocurre entre los $13-24$ meses de edad ${ }^{(17)}$.

La CIT inicialmente se desarrolla en superficies lisas, progresando rápidamente y produciendo un grave impacto en la dentición. El esmalte de los dientes deciduos tiene un grosor y calcificación menor que el de los dientes permanentes, lo cual favorece el rápido avance de las lesiones ${ }^{(18)}$.

Clínicamente, las lesiones de CIT-S se presentan en la superficie vestibular/palatina de incisivos superiores y siguen la secuencia eruptiva (con la inmunidad relativa de los incisivos inferiores protegidos por la lengua, el labio y la saliva); afectando posteriormente a los primeros molares superiores, primeros molares inferiores, caninos superiores y los segundos molares (17)

La típica forma de "media luna" o "semi-circular" de las lesiones en la superficie vestibular de los incisivos superiores coincide con la forma del margen gingival, donde se acumula la placa bacteriana madura. El "patrón muesca" conocido en la CIT-S ("notching decay pattern") descrito por Kotlow se presenta en infantes especialmente alimentados con lactancia materna nocturna a demanda sin higiene oral, estas lesiones representan el patrón de caries más precoz, ya que es el borde incisal el que es afectado ${ }^{(19)}$.

\section{Triada etiológica de Keyes}

Un factor de riesgo es cualquier rasgo, característica o exposición de un individuo que aumente la probabilidad de sufrir una enfermedad o lesión ${ }^{(20)}$. 
Los factores de riesgo de caries en niños son múltiples e incluyen la experiencia pasada de caries, información socio-demográfica y socioeconómica, condición médica, higiene oral, hábitos dietéticos, microorganismos orales, presencia de fluoruros y características del huésped, entre otros. Estos parámetros, que se pueden medir objetivamente, generan la determinación de nivel de riesgo subjetivo a través de diversas herramientas. El factor que predice de manera más precisa la probabilidad de lesiones cariosas en el futuro, es la experiencia pasada de caries ${ }^{(21)}$.

En 1960, Keyes estableció que la etiopatogenia de la caries dental obedece a la interacción de tres factores principales: huésped, microorganismo y sustrato; estos deben presentarse simultáneamente para que la caries se manifieste; si uno sólo de los componentes faltase, la caries no se desarrollaría, y si ésta ya existiese, se detendría. Este conjunto de agentes es conocido como Tríada Etiológica de Keyes (Figura 1). Los anteriores son considerados los factores primarios, 0 prerrequisitos para la iniciación de la caries. Asimismo, existen otros factores que pueden favorecer o dificultar la enfermedad, son los llamados factores secundarios; ejemplo de ellos son la composición de la saliva, edad del diente, morfología dentaria, concentración de fluoruros, frecuencia de la higiene bucal, número de comidas, etc.; todas estas están relacionadas en mayor proporción con algún componente de la triada, por lo que sería un indicador dentro de cada factor ${ }^{(15)}$.

En la Tabla 1 se puede observar estudios desde el 2010 en adelante, donde se resume los factores de riesgo considerados, agrupándolos de acuerdo a los componentes de la Triada Etiológica de Keyes.

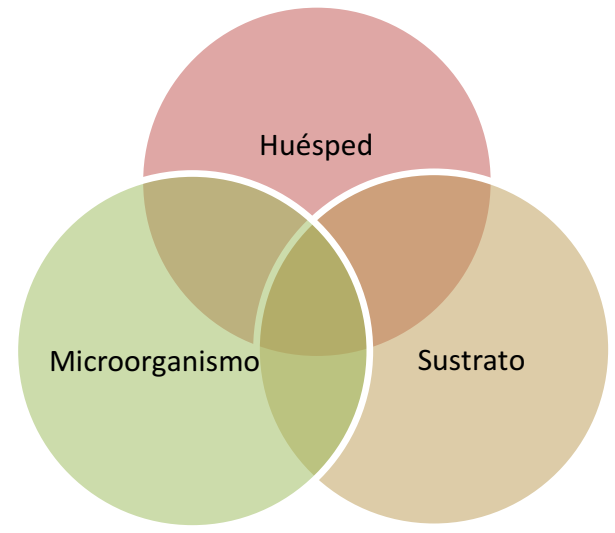

Figura 1. Esquema Triada Etiológica de Keyes: huésped, microorganismo y sustrato. Tres círculos se superponen: representan los factores primarios que conducen a la caries. Dado que los potenciales ) . patogénicos en cada uno de los tres parámetros principales pueden variar ampliamente, el impacto de cualquier agrupamiento es difícil de diagnosticar ${ }^{(14) .}$

Huésped. - Dentro de la Triada Etiológica de Keyes participa el huésped, quien es aquel que alberga la enfermedad de caries dental; el huésped no se refiere específicamente al ser humano, sino a todos aquellos factores que actúan en la fisiología del ser humano que permite que se desarrolle la enfermedad. En este caso se trata de la boca y los componentes que están dentro, la saliva y los dientes. La caries dental afecta directamente a los dientes y estos presentan características individuales en la persona, características anatómicas y de estructura, que permite que la enfermedad se inicie y avance de una forma particular. La saliva, por otro lado, posee propiedades protectoras contra la enfermedad, que en algunos casos puede estar disminuida y no cumplir adecuadamente su función ${ }^{(22) .}$

Diente. - Los dientes presentan tres particularidades relacionadas a favorecer el desarrollo de lesiones cariosas: proclividad, permeabilidad adamantina y su anatomía.

La proclividad se refiere a que ciertos dientes presentan superficies más propensas a la caries dental, la solubilización de minerales comienza en la parte más superficial del esmalte; a este nivel los prismas son ricos en fosfato de calcio y carbonatos de calcio, pero a medida que avanza la lesión al interior se va encontrando con presencia de carbonatos.

La permeabilidad adamantina disminuye con la edad, pierde la capacidad de incorporar moléculas a su estructura que mejoran sus propiedades físico-químicas. La proporción de los componentes del esmalte determinan la resistencia de este y, con ello, la velocidad de avance de la lesión.

La anatomía como zonas de contacto salientes o fosas y fisuras profundas, la disposición y la oclusión de los dientes, guardan estrecha relación con la aparición de lesiones cariosas, ya que favorecen la acumulación de placa y alimentos pegajosos, además de dificultar la higiene bucal ${ }^{(22) \text {. }}$

Saliva.- La saliva desempeña una función muy importante en la protección de los dientes frente a los ácidos. Actúa como una barrera que impide la difusión de los iones ácidos hacia el diente, así como el movimiento de los productos de la disolución del apatito hacia el exterior del diente. El flujo salival es estimulado por la cantidad de sacarosa de la boca, ocasionando la dilución y la deglución de la misma, evitando así el acúmulo de sustrato ${ }^{(22}$ 
Tabla 1. Revisión de artículos sobre factores de riesgo de CIT-S

\begin{tabular}{|c|c|c|c|c|c|c|c|c|c|c|c|c|c|c|c|}
\hline \multirow[b]{2}{*}{ Autor } & \multirow[b]{2}{*}{ Año } & \multicolumn{5}{|l|}{ Huésped } & \multicolumn{5}{|c|}{ Microorganismos } & \multicolumn{4}{|l|}{ Sustrato } \\
\hline & & Diente & Saliva & Inmunidad & Fluoruros & $\begin{array}{c}\text { Genética y } \\
\text { otros }\end{array}$ & $\begin{array}{c}\text { Premastica } \\
\text { los } \\
\text { alimentos }\end{array}$ & $\begin{array}{c}\text { Nivel de } \\
\text { Streptococos }\end{array}$ & $\begin{array}{c}\text { Nivel de } \\
\text { Lactobacilos }\end{array}$ & $\begin{array}{l}\text { Nivel de } \\
\text { Cándida }\end{array}$ & $\begin{array}{c}\text { Higiene } \\
\text { oral-placa } \\
\text { dental }\end{array}$ & $\begin{array}{l}\text { Lactancia } \\
\text { materna }\end{array}$ & $\begin{array}{l}\text { Lactancia } \\
\text { por } \\
\text { biberón }\end{array}$ & Azúcar & $\begin{array}{l}\text { Frecur } \\
\text { de cor }\end{array}$ \\
\hline \multicolumn{16}{|c|}{ Feldens et al 2010} \\
\hline Hashim et al & 2011 & & & & & & & & & & & & & & \\
\hline Kumar et al & 2011 & & & & & & & & & & & & & & \\
\hline Hughes et al & 2012 & & & & & & & & & & & & & & \\
\hline Schroth et al & 2013 & & & & & & & & & & & & & & \\
\hline Schroth et al & 2013 & & & & & & & & & & & & & & \\
\hline Bissar et al & 2014 & & & & & & & & & & & & & & \\
\hline Gilbert et al & 2014 & & & & & & & & & & & & & & \\
\hline Mahjoub et al & 2014 & & & & & & & & & & & & & & \\
\hline Nobile et al & 2014 & & & & & & & & & & & & & & \\
\hline Peltzer et al & 2015 & & & & & & & & & & & & & & \\
\hline Li et al & 2015 & & & & & & & & & & & & & & \\
\hline Bugra et al & 2016 & & & & & & & & & & & & & & \\
\hline Davidson et & & & & & & & & & & & & & & & \\
\hline al & 2016 & & & & & & & & & & & & & & \\
\hline Colombo et al & 2016 & & & & & & & & & & & & & & \\
\hline Mitrakul et al & 2016 & & & & & & & & & & & & & & \\
\hline Thomas et al & 2016 & & & & & & & & & & & & & & \\
\hline Xiao et al & 2016 & & & & & & & & & & & & & & \\
\hline Colombo et al & 2016 & & & & & & & & & & & & & & \\
\hline Kalpana et al & 2017 & & & & & & & & & & & & & & \\
\hline
\end{tabular}


En la saliva existen amortiguadores salivares que suelen mantener constante el $\mathrm{pH}$, son el bicarbonato, ácido carbónico y fosfato. Existe un $\mathrm{pH}$ crítico que es aquel en el cual la saliva está exactamente saturada con relación a la apatita del esmalte. El pH crítico se sitúa entre $5,2-5,5$ para la hidroxiapatita y 4,5 para la fluorapatita. Si el $\mathrm{pH}$ se reduce por debajo de este límite la saliva está hiposaturada y se inicia la perdida de mineral en la subsuperficie del esmalte. Las perdidas crónicas de mineral sumadas a lo largo de semanas y meses llegan a producir una lesión de caries visible que se observa como una mancha blanca. En esta fase la enfermedad puede detenerse sin necesidad de un tratamiento restaurador, favoreciéndose la remineralización de la lesión, aunque no se pueda conseguir una desaparición completa de la mancha blanca ${ }^{(22)}$.

Fluoruros. La medida de mayor impacto para el control del desarrollo de la caries dental ha sido el uso de flúor. Aunque su uso aislado no impide la aparición de la caries, sí reduce su progresión. El declive mundial de la manifestación de esta enfermedad ha sido atribuido al uso de una o varias formas de utilización del flúor.

En vista de que el flúor interfiere en la dinámica del proceso de caries, debe considerarse más importante su presencia constante en el medio ambiente bucal en lugar de su incorporación al diente. El flúor no interfiere en los factores responsables de la enfermedad de la caries pero reduce la manifestación de su progresión (23).

Inmunización. La saliva presenta factores protectores ante la caries dental. Entre estos tenemos a la lisozima, lactoferrina, peroxidasa salival, mieloperoxidasa, tiocianato, la inmunoglobulina A ( $\lg A)$ salival e inmunoglobulina $G$ sérica. En los infantes estos factores están disminuidos porque su sistema inmune aún está inmaduro.

La respuesta inmunitaria de la $\lg A$ representa la primera línea de defensa inmune adaptativa contra los Streptococcus mutans, bloqueando las adhesinas microbianas y potencialmente reduciendo la colonización oral con esta microflora cariogénica. También se ha demostrado que la lgA salival mejora la actividad de varias enzimas tales como lactoferrinas y lisozimas ${ }^{(24) .}$

Genética y otros. Se estima que la contribución genética a la caries dental es de aproximadamente un $40 \%$. Los factores predisponentes a la caries dental son sumamente variados lo que hace difícil que intervenga un solo gen. Una alternativa para identificar los genes candidatos como los principales es la revisión del genoma, ya que de otra forma no se podría asociar al proceso de caries dental ${ }^{(22) \text {. }}$

\section{Microorganismos}

Los principales microorganismos causantes de la caries son los estreptococos del grupo mutans, especialmente el Streptococcus mutans y el Streptococcus sobrinus. Estos patógenos son capaces de colonizar la superficie del diente y producir ácidos en velocidad superior a la capacidad de neutralización de la biopelícula en un ambiente bajo el pH crítico (menor de 5,5), permitiendo la disolución del esmalte ${ }^{(25) .}$

El reservorio principal de donde el lactante adquiere los Streptococcus mutans es la madre (transmisión vertical) (26); actualmente también se puede considerar a las nanas y abuelas que son responsables del cuidado diario del infante (5). La transmisión vertical de madre a niño se produce por transferencia de saliva infectada al besarlo en la boca, o con mayor frecuencia, al humedecer con saliva la tetina del biberón o el "chupón", o probar y premasticar los alimentos en la cucharita del pequeño antes de servirlos $(11,27,28)$. La colonización de microorganismos por transmisión vertical se relaciona con varios factores como la magnitud del inóculo, la frecuencia de inoculaciones de pequeñas dosis y un mínimo de dosis infectiva. Los niños con madres portadoras de altas concentraciones de Streptococcus mutans, adquieren esos microorganismos tempranamente $y$ en niveles mayores. La frecuencia de la infección infantil es nueve veces mayor cuando las madres presentan cantidades de Streptococcus mutans que exceden 105 unidades formadoras de colonias por mililitro de saliva ${ }^{(27) \text {. }}$

También se han descrito transmisiones horizontales en cunas de guarderías e intrafamiliares. La gravedad de la CIT está directamente relacionada con la precocidad de la instalación de los estreptococos del grupo mutans en el niño ${ }^{(29,30) .}$

Otros microorganismos involucrados, como los lactobacilos, se asocian a la progresión de una lesión ya instalada, y no a la iniciación de la caries propiamente dicha (Tabla 2) ${ }^{(25) \text {. }}$

Ma et al. (2015) estudiaron los perfiles microbianos orales en niños con CIT-S, encontrando 379 especies bacterianas, entre ellas varios géneros, incluyendo Streptococcus, Porphyromonas y Actinomyces, estos están fuertemente asociados con CIT-S y pueden ser biomarcadores potenciales de la caries dental en la dentición primaria ${ }^{(31)}$. 
Thomas et al. (2016) indican que en la primera infancia, los niños son más susceptibles a la colonización microbiana oportunista en la cavidad oral debido al sistema inmunológico inmaduro y a que su microflora no está completamente establecida. Actualmente se propone un probable papel del hongo Candida albicans en la etiopatogenia de la caries dental, así como también en CIT-S. Su estudio demostró que hubo un aumento muy significativo en el recuento de Candida albicans en niños con CIT-S en comparación con los niños sin caries ${ }^{(32) .}$

La formación de la placa dental constituye el primer paso para el establecimiento de los microorganismos patógenos en la cavidad bucal. El grado de cariogenicidad de la placa dental depende de varios factores: la localización especifica de la masa de microorganismos, el número de microorganismos concentrados en ciertas áreas no accesibles a la higiene y la producción de ácidos ${ }^{(22)}$.

Tabla 2. Principales bacterias que intervienen en la formación de caries dental

\begin{tabular}{|c|c|}
\hline Bacterias & Características \\
\hline $\begin{array}{l}\text { Streptococos } \\
\text { mutans }\end{array}$ & $\begin{array}{l}\text { - Producen grandes cantidades } \\
\text { de polisacáridos extracelulares } \\
\text { que permiten una gran } \\
\text { formación de placa. } \\
\text { - Producen una gran cantidad de } \\
\text { ácido a bajos niveles de pH. } \\
\text { Rompen algunas } \\
\text { glicoproteínas salivales } \\
\text { importantes para impedir las } \\
\text { etapas de desarrollo inicial de }\end{array}$ \\
\hline Lactodacmus & $\begin{array}{l}\text { - Aparece cuando existe una } \\
\text { frecuente ingesta de } \\
\text { carbohidrato }\end{array}$ \\
\hline Actynomices & $\begin{array}{l}\text { cariosas radiculares. } \\
\text { - Raramente inducen caries de } \\
\text { esmalte. } \\
\text { Producen lesiones de } \\
\text { progresión más lenta que los } \\
\text { otros microorganismos. }\end{array}$ \\
\hline
\end{tabular}

Modificado de Henostroza (2007) ${ }^{(22)}$.

\section{Sustrato}

Existe suficiente evidencia para afirmar que los azúcares son los principales elementos de la dieta diaria que influyen en la prevalencia y el avance de las lesiones de caries dental (Tabla 3) ${ }^{(33-35) .}$

La sacarosa es el alimento cariogénico más importante y más ampliamente utilizado por el hombre. Tiene el poder de transformar alimentos no cariogénicos $y$ anticariogénicos en cariogénicos. Los demás azúcares involucrados en la cariogénesis son la glucosa y la fructosa, encontrados en la miel y en las frutas. Una simple exposición a los alimentos cariogénicos no es un factor de riesgo para la caries, sino el frecuente y prolongado contacto de estos sustratos con los dientes
$(21,36)$.

Feldens et al. (2010) estudiaron las prácticas de alimentación que representan factores de riesgo para la aparición de CIT-S en niños de 4 años. Encontraron una asociación significativa entre la CIT-S y la lactancia materna $(R R=1,97 ;$ IC $95 \%=1,45-2,68)$, la alta densidad de azúcar ( $R R=1,43 ;$ IC $95 \%=1,08-1,86)$, así como el número de comidas y meriendas ( $R R=1,42$; IC del $95 \%$ $=1,02-1,97)^{(33)}$.

Bissar et al. (2014) encontraron que la lactancia materna durante más de 12 meses y el uso del biberón en cama son factores de riesgo significativos en la aparición de CIT-S ${ }^{(37)}$

Tabla 3. Factores relativos al sustrato

- $\quad$ Tipo de carbohidrato

- Cantidad total de carbohidratos fermentables

- $\quad$ Adhesividad de retención.

- Compuestos protectores adicionales a la dieta (flúor, calcio, fosfatos, proteínas, grasas).

- Acidez del alimento.

- Secuencia de ingesta de alimentos

Modificado de Henostroza (2007) ${ }^{(22)}$.

\section{Conclusiones}

$\checkmark \quad$ No se encontraron investigaciones sobre la premasticación de alimentos como factor de riesgo para CIT-S, pero si como factor de riesgo de CIT.

$\checkmark \quad$ El nivel de Streptococos mutans es un factor de riesgo significativo para CIT-S en todas las investigaciones revisadas. 
$\checkmark$ Se encontraron pocos estudios del nivel de Lactobacilos y Candida como factor de riesgo de CIT-S, falta sustento científico para determinarlos como factores de riesgo

$\checkmark$ Se encontraron varias investigaciones que estudiaron a la higiene oral o placa bacteriana como factor de riesgo de CIT-S, pocos indicaron significancia.

$\checkmark \quad$ La mayoría de investigaciones avalan que la lactancia materna y por biberón son factores de riesgo significativos para CIT-S.

\section{Recomendaciones}

A nivel clínico, llama la atención las características particulares de la presentación única de la enfermedad, lo que sugiere que existen factores causales específicos para su aparición y desarrollo. A nivel etiológico, se debe comprender todos los factores patológicos que participan en esta enfermedad en la infancia, brindar pautas personalizadas para cada paciente, basadas en el riesgo individual del niño.

La evaluación del riesgo de caries debe incluir todos los factores primarios que se desarrollan dentro de la Triada Etiología de Keyes, esta propuesta nos brinda un orden grupal donde podemos incluir a la gran mayoría de factores de riesgo, siempre dejando en claro que no se toma en cuenta los factores sociales que también pueden afectar de alguna manera.

A pesar del gran avance en el conocimiento de la CIT$S$, en los últimos años, aún se necesita mayor comprensión de los factores de riesgo primarios, con el fin de determinar las estrategias de prevención específicas en estos pacientes.

\section{REFERENCIAS BIBLIOGRÁFICAS}

1. Daly B, Watt R, Batchelor P, Treasure E. Essential Dental Public Health. New York: Oxford University Press. 2002.

2. Bernabé E, Delgado-Angulo $K$, Sánchez-Borjas $P$. Resultados de un sistema para la vigilancia de caries de la infancia temprana . Rev Med Hered. 2006;17(4):227-33.

3. Zaror Sánchez C, Pineda Toledo P, Orellana Cáceres JJ. Prevalencia de Caries Temprana de la Infancia y sus Factores Asociados en Niños Chilenos de 2 y 4 Años. Int J Odontostomatol. 2011;5(2):171-7.

4. Villena Sarmiento R, Pachas Barrionuevo F, Sánchez Huamán Y, Carrasco Loyola M. Prevalencia de caries de infancia temprana en niños menores de 6 años de edad, residentes en poblados urbano marginales de
Lima Norte. Rev Estomatológica Hered [Internet]. 2014;21(2):79. Disponible en :http://www.upch.edu.pe/vrinve/dugic/ revistas/index.php/ $\mathrm{REH} /$ article/view/237

5. Achahui Arauco P, Albinagorta MJ, Arauzo Sinchez CJ, Cadenillas Sueldo AM, Céspedes Martinez GP, Cigüeñas Raya EM, et al. Caries de Infancia Temprana: diagnóstico e identificación de factores de riesgo. Odontol Pediatr. 2014;13(2):119-37.

6. Dentistry AA of P. Policy on early childhood caries (ECC): classifications, consequences, and preventive strategies. Pediatr Dent. 2011;30(7):40-3.

7. Tinanoff N. Introduction to the Conference: Innovations in the Prevention and Management of Early Childhood Caries. In: Pediatric Dentistry. 2004. p. 198-9.

8. Folayan $\mathrm{MO}$, Kolawole $\mathrm{KA}$, Oziegbe $\mathrm{EO}$, Oyedele $\mathrm{T}$, Oshomoji O V, Chukwumah NM, et al. Prevalence, and early childhood caries risk indicators in preschool children in suburban Nigeria. BMC Oral Health [Internet]. 2015;15(1):72. Available from: http://bmcoralhealth.biomedcentral.com/articles/10.1186/ s12903-015-0058-y

9. Fan C, Wang W, Xu T, Zheng S. Risk factors of early childhood caries among children in Beijing: a case-control study. BMC Oral Health [Internet]. 2016;16(1):98. Disponible en: http://bmcoralhealth.biomedcentral. com/articles/10.1186/s12903-016-0289-6

10. Custodio-Lumsden CL, Wolf RL, Contento IR, Basch CE, Zybert PA, Koch PA, et al. Validation of an early childhood caries risk assessment tool in a low-income Hispanic population. J Public Health Dent [Internet]. 2016;76(2):136-42.Disponible en: http://doi.wiley.com/10. 1111/jphd.12122

11. van Palenstein Helderman, WH Soe W, Van't Hof M. Risk Factors of Early Childhood Caries in a Southeast Asian. J Dent Res. 2006;85(1):85-8.

12. Carmona Arango LE, Gonzales Martinez F, Hernández Blanco JF, Paternina Sierra KP. Prevalencia de caries en infancia temprana y factores asociados en niños de hogares comunitarios en la ciudad de Cartagena. 2013:191.

13. Levine J, Wolf RL, Chinn C, Edelstein BL. MySmileBuddy: An iPad-Based Interactive Program to Assess Dietary Risk for Early Childhood Caries. J Acad Nutr Diet [Internet]. 2012;112(10):1539-42. Available from: http://dx.doi.org/10.1016/j.jand.2012.06.010

14. Keyes PH. Present and future measures for dental caries control. J Am Dent Assoc [Internet]. 1969;79(6):1395404. Available from: http://dx.doi.org/10.14219/jada.archive.1969.0037

15. Keyes $P$. Recent advances in dental caries research. Bacteriological findings and biological implications. Int Dent J. 1962;12(4):443-64. 
16. American Academy. Policy on early childhood caries (ECC): Classification, consequences, and preventive strategies. Oral Heal Policies. 2014;37(6):50-2.

17. Edelstein BL, Chinn $\mathrm{CH}$, Laughlin RJ. Early Childhood caries: definition and epidemiology. En: Berg JH, Slayton RL, editores. Early Childhood Oral Health. 1a ed. lowa: Wiley-Blackwell; 2009: 18-49.

18. American Academy of Pediatric Dentistry A. Guideline on Caries-risk Assessment and Management for Infants, Children, and Adolescents. Clin Guidel Ref Man 2015-2016. 2015;37(6):132-9.

19. Kotlow LA. The influence of the maxillary frenum on the development and pattern of dental caries on anterior teeth in breastfeeding infants: prevention, diagnosis, and treatment. J Hum Lact. 2010;26(3):304-8.

20. OMS. http://www.who.int/topics/risk_factors/es/.

21. Mejàre I, Axelsson S, Dahlén G, Espelid I, Norlund a, Tranæus S, et al. Caries risk assessment. A systematic review. Acta Odontol Scand [Internet]. 2013;6357(January):1-11. Available from: http://www.ncbi.nlm.nih.gov/pubmed/23998481

22. Henostroza G. Caries dental: Principios y procedimientos para el diagnóstico. Lima: Editorial Universidad Peruana Cayetano Heredia; 2007.

23. Cury J. Uso do Flúor e Controle da Cárie como Doença. In: Baratieri L, editor. Odontologia Restauradora - Fundamentos e Possibilidades. Sao Paulo: Santos; 2001. p. 33-68.

24. Colombo NH, Pereira JA, Da Silva MER, Ribas LFF, Parisotto TM, Mattos-Graner RDO, et al. Relationship between the $\lg A$ antibody response against Streptococcus mutans $\mathrm{GbpB}$ and severity of dental caries in childhood. Arch Oral Biol [Internet]. 2016;67(2016):22-7. Available from: http://dx.doi.org/10.1016/j.archoralbio.2016.03.006

25. Seow WK. Biological mechanisms of early childhood caries. Community Dent Oral Epidemiol [Internet]. 1998;26(14):8-27. Disponible en: http://search.ebscohost.com/login.aspx?direct=true\&d $b=d d h \& A N=12014678 \&$ lang $=p t-b r \&$ site=ehost-live

26. Heymann D. El Control de las Enfermedades Transmisibles. Informe Oficial de la Asociación. Estadounidense de Salud Pública. Washington, DC, EUA: $18^{\circ}$; 2005. p. 2005

27. Berkowitz R. Mutans Streptococci: Adquisition and Transmission. Pediatr Dent. 2006;28(2):106-9.

28. Figueiredo W, Ferelle A, Issao M. Odontología para el Bebé. Actualidades Médico Odontológicas Latinoamérica. Caracas, Venezuela; 2000.

29. Mattos-graner RO, Li Y, Caufield PW, Duncan M, Smith DJ. Genotypic Diversity of Mutans Streptococci in Brazilian Nursery Children Suggests Horizontal Transmission Genotypic Diversity of Mutans
Streptococci in Brazilian Nursery Children Suggests Horizontal Transmission. J Clin Microbiol. 2001;39(6):2313-6.

30. Doméjean S, Zhan L, DenBesten P, Stamper J, Boyce W, Featherstone J. Horizontal Transmission of Mutans Streptococci in Children. J Dent Res. 2010;89(1):51-5.

31. Ma C, Chen F, Zhang $Y$, Sun $X$, Tong $P$, Si $Y$, et al. Comparison of oral microbial profiles between children with severe early childhood caries and caries-free children using the human oral microbe identification microarray. PLoS One. 2015;10(3):1-12.

32. Thomas A, Mhambrey S, Chokshi K, Chokshi A, Jana S, Thakur S, et al. Association of Oral Candida albicans with Severe Early Childhood Caries - A Pilot Study. J Clin Diagnostic Res [Internet]. 2016;10(8):109-12. Available from: http: //jcdr.net/article_fulltext.asp?issn=0973709 $x \&$ year $=2016 \&$ volume $=10 \&$ issue $=8 \&$ page $=Z C 109 \&$ iss $n=$ 0973-709x\&id=8357

33. Feldens CA, Giugliani ERJ, Vigo Á, Vítolo MR. Early feeding practices and severe early childhood caries in four-year-old children from southern Brazil: A birth cohort study. Caries Res. 2010;44(5):445-52.

34. Peltzer K, Mongkolchati A. Severe early childhood caries and social determinants in three-year-old children from Northern Thailand: a birth cohort study. BMC Oral Health [Internet]. 2015;15(1):108. Available from: http://bmcoralhealth.biomedcentral.com/articles/10.1186/ s12903-015-0093-8

35. Hashim R, Williams S, Thomson WM. Severe early childhood caries and behavioural risk indicators among young children in Ajman, United Arab Emirates. Eur Arch Paediatr Dent. 2011;12(4):205-10.

36. Harris R, Nicoll AD, Adair PM, Pine CM. Risk factors for dental caries in young children: A systematic review of the literature. Community Dent Health. 2004;21(1 SUPPL.):71-85.

37. Bissar A, Schiller P, Wolff A, Niekusch U, Schulte AG. Factors contributing to severe early childhood caries in south-west Germany. Clin Oral Investig. 2014;18(5):1411-8. 